\title{
A Circles Model to Measure and Predict Project Success
}

\author{
Mohammadreza Parsanejad ${ }^{1}$, Hiroaki Matsukawa ${ }^{1}$, and Tomoichi Sato ${ }^{2}$ \\ ${ }^{1}$ Graduate School of Science and Technology, Keio University, Tokyo, Japan \\ 2 JGC Corporation, Tokyo, Japan
}

\begin{abstract}
Measuring project success is a challenging issue among researchers and practitioners, because of its multidimensional properties. During the last three decades, numerous researchers have tried to define and illustrate its dimensions. But still there is no general agreement about it. To measure and predict success of a project, we need to take into account all project stages including utilization stage of the product of a project in an integrated approach. In this paper we try to introduce a novel model to measure project success considering selection, execution and utilization stages. The model is based on the deviations from the goal of each stages. And the amount of success in this model which we call the "Circles Model", is the magnitude of proximity to the final goal. We apply it to an example and measure its success quantitatively. Lastly we discuss four extensible directions for future studies to apply the model on high performance projects, multi stakeholders space, program and portfolio domain and finally to explore the influence of the performance of each stage on the performance of the next stages.
\end{abstract}

Key Words : Project success, Measurement, Circles model, Deviation

\section{Introduction}

Every day thousands of projects are being defined, initiated, planned, executed, controlled and closed out. They create products or services for the customers and produce values for their stakeholders (PMI, 2008). But yet their success are not easily measured and if measured rarely agreed between conflicting stakeholders (Ika, 2009). Measuring project success is a sophisticated action in which many parameters are incorporated. Each project has different stakeholders with different viewpoints and different phases during the project life cycle has different evaluation criteria. These diversities amplify the difficulty of project evaluation. To overcome these difficulties we need to introduce an integrated model to measure success of the project considering three stages i.e. selection, execution and utilization stage. In the following sections at first we take a look at literature, then introduce the model and apply it to a measure success of the project. At the end we discuss some directions that extend application of the model to a wide range of situations.

\section{Literature Review}

Looking at the literature we found that there is a significant revolution in the measuring project success at the end of the $80 \mathrm{~s}$. Dewit (1988) in his famous paper distinguishes between project management success and project success. The division is made based on the inherent difference between execution and utilization stages. When a project is delivered on time, under budget and according to the desired specification, it will be considered as successful project management, not project success as

Corresponding Author: Mohammadreza Parsanejad

Faculty of Science and Technology, Keio University, Tokyo, Japan

Parsanejad@a7.keio.jp

(Received February 12, 2013)

(Revised May 8, 2013)

(Accepted May 29, 2013) a whole. Inversely there are situations that project management failed to meet Iron Triangle, but still considered as successful (Atkinson 1999). There are lots of examples in this issue such as London's Millennium Dome and Sydney Opera Hall. Subsequently not only project execution stage should be evaluated but also its functionality or satisfaction of its end user. Distinguishing project management success from product success clarifies this conflict. Indeed project success is summation of project management and product success (Baccarini, 1999).

Unfortunately aggregating the two concepts of project and project management success have not solved all problems and researchers believe that the concept is still ill defined without deep meaning (Hyvari, 2006). One of the major reasons of its weakness is the existence of Type II errors (Atkinson, 1999) that is forgotten aspect of measurement which should be take into account. In a comprehensive survey on more than 170 papers, Parsanejad and Matsukawa (2011) show that still there are some hidden aspects i.e. Type II errors in project success evaluation. They believe that researchers working on the project success discipline in an evolutionary attempt extend the concept of project management success toward project success by considering utilization stage of project's product. Then they discover another extension toward measuring program and portfolio success as a developed version of single project success measurement. They call these extensions as Forward, Horizontal extension. Afterward they demonstrate another aspect of story that almost forgotten or never discussed in the context of project success. That is project selection success. Although project selection is a well-known field among researchers but few studies consider it to evaluate success of a project. Selection of a project is as important as of others stages, to the point that Munns and Bjeirmi (1996) allocate an extraordinary emphasis to the role of selection stage and state that "The right project will succeed almost without the success of project management". 
There are few studies considering all three stages (Selection, Execution and Utilization) in the process of measuring project success. Yu et al.(2005) incorporate different measurement criteria to a single one, and develop a value centered concept to measure success of the project from client's viewpoint. Although they build a new method to evaluate the project success as a whole but they do not clearly clarify influence of each stages i.e. selection, execution and utilization, on the total project success. Khang and Moe (2008) address four success criteria for measuring project selection success and then combine them to the execution and utilization stages, but their model is just putting criteria relating to three stages in a table not integrating them. And they do not propose any quantitative solution to evaluate project success. The only paper that substantially integrates all three stages alongside detailed definition of each part is Pillai et al.(2002). They define some criteria for each stage trying to make a model to measure project success in $R \& D$ projects, but they fail to calculate effect of performance of each stage on the performance of the next stages. When a project is selected inappropriately, its execution and utilization will be affected by improper selection decision. On the other side of spectrum there are projects which are carefully selected and there are many potential opportunities behind them. In these projects even if project management fails to meet time and cost threshold, they will be succeed partially. The Sydney opera hall is a suitable example for this specific situation. The method proposed by Pillai et al.(2002) could not explain such situations.

In order to integrate the three stages and also to capture and calculate the influence of performance of each stage on the next stages, we aim to make a model to quantitatively measure and predict project success. In the next section we introduce our model and demonstrate its dimensions.

\section{Circles Model}

\subsection{Model Configuration}

The main idea of the model we aim to propose is based on deviation from goals not meeting them. Although these two concepts are two sides of a single notion but we primarily measure the deviation from goals and then calculate meeting of the goals based on it. In the project environment deviations are due to the mistakes in decision makings during selection, execution and utilization stages. All deviations happened during these three stages are aggregated and then construct the total amount of the deviation. Deviations from final goal of a project i.e. end user satisfaction, might happen during each of the three stages. Deviation through selection stage not only itself is parts of total deviation but also has a negative effect on the performance of the execution and utilization stages and consequently causes some deviations through them. Identically deviation within execution stage increases total deviation directly meanwhile influences indirectly and negatively on the performance of its next stage (utilization). And the utilization stage as the final stage if deviate, purely increase the total deviation and does not has any other side effect. As a result there are six deviations, three of them are pure deviations during three stages, and the three others are influenced deviations caused by deviations of previous stages. Summation of these deviations creates the total amount of deviation from the final goal.
On the other hand, different stages in projects under different circumstances will have different amount of influence on the total deviation. The influence of each stage fundamentally depends on the amount of relative importance of stages on reaching the final goal. In a project with high amount of sensitivity to selection stage, for example $R \& D$ projects, the relative importance of selection stage is higher than other two stages and therefore even small amount of deviation in this stage will produce huge amount of deviation from final goal. In contrary the power of an organization in a market might establish an environment in which any project selected and initiated by that company will acquired high amount of success. The latter happens when there is monopoly or when there is a deep loyalty to a famous brand even if the product of project is not too useful for the customers. In this case the relative importance of selection stage is less than the first example.

These interrelationships are shown in the Fig 1, where $a_{1}, a_{2}$ and $a_{3}$ indicate the deviation of selection, execution and utilization stages from reaching to the goal, respectively, and the $i_{1}$, $i_{2}$ and $i_{3}$ show the relative importance of three stages at reaching that goal. The $a_{1}, a_{2}$ and $a_{3}$ could be any number between 0 and +180 degrees. Positive numbers show the existence of the deviation in the processes; whereas zero shows condition where there is no deviation. In this paper we do not consider the negative amounts which happens in the case of very high performance projects. Relative importance numbers $i_{1}, i_{2}$ and $i_{3}$, which are sort of weights allocated to each stage, should be numbers between zero and one with total summation of one.

We consider three circles as three project stages, placed within each other. Circles are concentric and the center shows the start of selection process of the project. When the project idea progress and project is selected, first stage finishes and as a result an arrow is being drown from the circles' center to a point on the first circle. The position of this point is depending on the amount of deviation from the goal $\left(a_{1}\right)$ due to the mistakes occurred in the selection stage. Similar procedures take place for the execution as well as utilization stage.

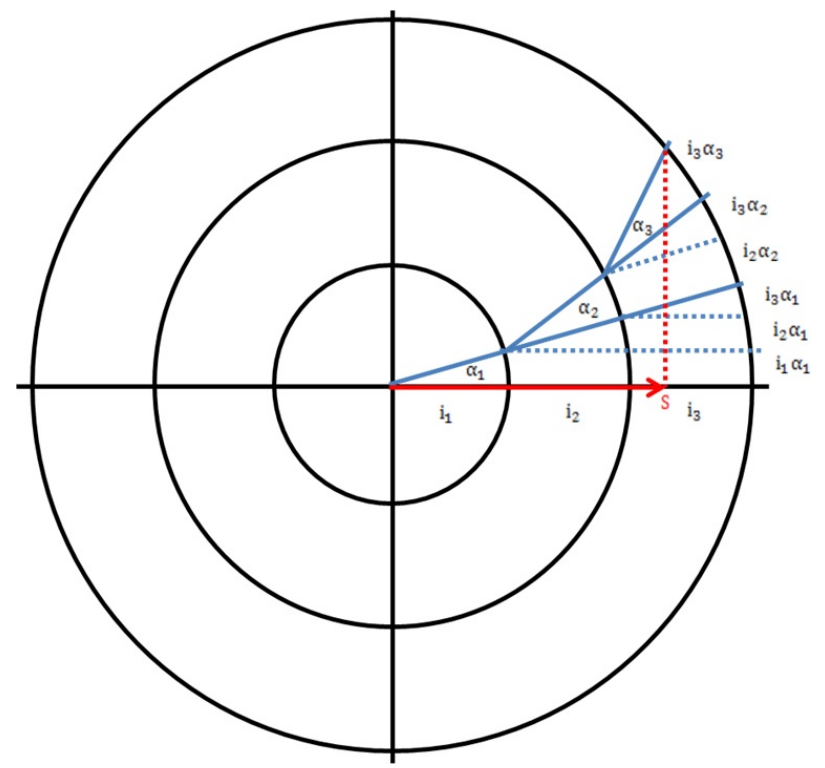

Fig. 1 Model Configuration

We could estimate the total amount of deviation (D) using simple calculation as below; 


$$
\begin{aligned}
& D=\left(i_{1}+i_{2}+i_{3}\right) a_{1}+\left(i_{2}+i_{3}\right) a_{2}+i_{3} a_{3} \\
& D=i_{1} a_{1}+i_{2} a_{1}+i_{3} a_{1}+i_{2} a_{2}+i_{3} a_{2}+i_{3} a_{3}
\end{aligned}
$$

This is an approximation of deviation because of the noncentrality of three arcs corresponding three stages. Therefore the model has a specific amount of error. Later we will show that this error is low for lower amounts of deviations. The amount of error is enough small to we continue the calculation based on these formula.

The above equation has six elements. As mentioned before three of them $\left(i_{1} a_{1}, i_{2} a_{2}\right.$ and $\left.i_{3} a_{3}\right)$ show the pure deviation from final goal caused by each stage. These terms show the direct effect of managerial or functional mistakes on the total success. Furthermore $i_{2} a_{1}$ and $i_{3} a_{1}$ illustrate the impact of deviation of selection stage on the total deviations through the next two stages. And finally $i_{3} a_{2}$ is the influence of deviation of second stage on the third stage. These terms illustrate the ability of the model to measure interrelationship between different stages and influence of the performance of each stage on the performance of its following stages. To sake of simplicity we label these elements as follow;

$$
D=D_{1}+D_{12}+D_{13}+D_{2}+D_{23}+D_{3}
$$

After measuring the amount of total deviation (D) the total amount of success are calculated based on the below equation;

$$
S=\operatorname{Cos}(D)
$$

We use cosines to determine the amount of success, because it significantly analogous to the concept of success in our model. It maps the position of the final point on the utilization stage to a line that connects project start to project goal. The mapped amount that is the shadow of the actual line shows magnitude of meeting the goal. The cosine function has also other advantage. It maps the amount of deviation onto a number between -1 and 1 . Therefore we are able to show not only the success but also the failure of a project. The negative numbers $(-1 \leq S<0)$ show the amount of failure and positive ones $(0<S \leq 1)$ show the amount of success.

\subsection{Model Assumptions}

We have some assumptions to build the model. At first we consider that a project has a start point and a finish point. Based on the PMBOK definition, a project is a temporary endeavor undertaken to create a unique product, service, or result. The temporary nature of projects indicates a definite beginning and end. The end is obtained when the project's objectives are achieved or when the project is terminated because its objectives will not or cannot be met, or when the need for the project no longer exists (PMI, 2008). It can be seen that PMBOK guide, defines start and end of the project as start and end of execution stage. But we need an extended viewpoint to analysis the project success. But we need a wider viewpoint that consider project life cycle. According to PMBOK guide project phases are divisions within a project where extra control is needed to effectively manage the completion of a major deliverable. Project phases constructs project life cycle that is a subsidiary of the product life cycle (Jugdev and Muller, 2005). This is what we need.
Based on the Japanese standard of project management so called Project and Program Management Standard (P2M), the projects have three parts i.e. scheme, system and service, which correspond to the three phases of our model, selection, execution and utilization respectively.

We use the definition of P2M standard and extent the concept of project success to three stages to cover the influence of all three stages on the total project success. We assume that start point of project selection stage is when an idea sparks in the owner's mind, or when the owner should choose one project from a portfolio of projects. The end point of project selection stage is when the project is selected and is going to be initiated. Start and finish point of execution stage is exactly same as PMBOK definition as mentioned before. And finally the start and end point of utilization stage is from delivery of product to the end of product lifecycle.

We also assume that the evaluation of project success is done on one of the three point, end of selection stage, end of execution stage, and any point on the utilization stage. This is due to the reduction of facing complexities in case of evaluation in the middle of first and second stage.

The final assumption of model is about influence of the mistakes in previous stages on the next stages. Obviously the final stage i.e. utilization does not have any impact on the others, because execution and selection stages have already done. Using same logic utilization stage does not effect on the selection stage. Therefore three influenced deviations are:

- Effect of selection stage on the performance of execution stage $\left(D_{12}\right)$

- Effect of selection stage on the performance of utilization stage $\left(D_{13}\right)$

- Effect of execution stage on the performance of utilization stage $\left(D_{23}\right)$

In our model we suppose that the three influenced deviations are equal to $a_{1} i_{2}, a_{1} i_{3}$ and $a_{2} i_{3}$. For example $D_{12}$ is equal to,

$$
D_{12}=a_{1} \times i_{2}
$$

It means that the amount of influence of mistakes during stage 1 on the deviation created in stage $2\left(D_{12}\right)$, is a function of amount of deviation is stage $1\left(a_{1}\right)$, multiplying the relative importance of stage $2\left(i_{2}\right)$.

To analysis this assertion we start from the zero conditions. In case of zero deviation in stage $1\left(a_{1}=0\right)$ the amount of influenced deviation obviously would be zero $\left(D_{12}=0\right)$. If everything is exactly true in prior stages, they will not create any deviation in the subsequent stages. In this condition project manager should not be worry about the previous stages and it is enough to focus on his/her effectiveness in the current stage (execution stage). The extent to which project deviation from its final goal increase, it will negatively influence on the performance of next steps. The meaning of this status is that consuming the resources i.e. time, money and human resources, during execution of a inappropriately selected project, is itself kind of deviation $\left(0<D_{12}\right)$, even though its execution is exactly on time, under budget and without any deviation $\left(D_{2}=0\right)$.

On the other hand the higher relative importance of the second stage $\left(i_{2}\right)$, the higher influenced deviation $\left(D_{12}\right)$. Because 
the project will consume more resources in the way that is not exactly on the direction of the final goal (for example: end user satisfaction). If this relative importance are equal to zero $\left(i_{2}=0\right)$, the amount of influenced deviation again would be zero $\left(D_{12}=0\right)$. The zero important tasks never accept influence nor create deviation, because they never consume resources.

\subsection{Relative importance of three stages}

The question arises about the method of measuring deviations $\left(a_{1}, a_{2}\right.$ and $\left.a_{3}\right)$ and relative importance $\left(i_{1}, i_{2}\right.$ and $\left.i_{3}\right)$. In the next two steps we discuss how to calculate them.

To measure relative importance of each stage we need to understand their inherent properties. $i_{1}, i_{2}$ and $i_{3}$ could be captured by a simple questionnaire asking about relative importance of three stages at meeting final goal, for example end user satisfaction. This method of evaluation is simple but a little bit confusing for respondents. Because experts as assessors will be confused about such general question. A more complex but practical way to capture the amount of relative importance is to calculate it through the concept of risk. In this context the extent to which the risk of a stage is high, it is hard to perform and also it has higher influence on the value acquisition and therefore it has higher importance. As a result the relative importance of each stage could be shown as a function of risk. Having a project with three stages, the risk of stage 1, 2 and 3 are $r_{1}, r_{2}$ and $r_{3}$, respectively.

In such project, after stage 3 project is expected to attain the value V. Considering the meaning of the risk of project, at the beginning of stage 3,2 and 1 the expected value is $\left(1-r_{3}\right) V$, $\left(1-r_{2}\right)\left(1-r_{3}\right) V$ and $\left(1-r_{1}\right)\left(1-r_{2}\right)\left(1-r_{3}\right) V$, respectively. In this context value improvement by stage 1 would be the difference between values expected at the end of stage minus values expected at start of that stage. This specific amount for stage 1 is calculated as below;

$$
\left(1-r_{2}\right)\left(1-r_{3}\right) V-\left(1-r_{1}\right)\left(1-r_{2}\right)\left(1-r_{3}\right) V=r_{1}\left(1-r_{2}\right)\left(1-r_{3}\right) V(6)
$$

The amount of value improvement for stage 2 and 3 are calculated similarly as below;

$$
\begin{aligned}
& \left(1-r_{3}\right) V-\left(1-r_{2}\right)\left(1-r_{3}\right) V=r\left(1-r_{3}\right) V \\
& V-\left(1-r_{3}\right) V=r_{3} V
\end{aligned}
$$

The amount of value improvement for each stage illustrate the amount of importance of that stage. To find the relative importance numbers it is enough to we divide the value improvement of each stage by the total expected value of project $\mathrm{V}$. In this manner we easily delete the amount of $\mathrm{V}$, and therefore the relative importance numbers for stages 1,2 and 3 are $r_{1}\left(1-r_{2}\right)\left(1-r_{3}\right), r\left(1-r_{3}\right)$ and $r_{3}$ respectably.

There are three unknown parameters i.e. $r_{1}, r_{2}$ and $r_{3}$. The risk of each stage could be captured by asking experts through questionnaires or interview. For activities or stages that are already finished we should ask about the amount of risk of performing all the activities including past stages. About past stages, we ask which level of risk we faced with. And about upcoming stages the question should be in a format in which we ask about the amount of risk that we might face with in the future.

\subsection{Relative importance of three stages}

Deviations from the goal in each stage could be evaluated by more than one criterion. Each project has its own criteria and each criterion would has different weights in different projects. Therefore for each project we design two types of questionnaires for each of three stages. The first type captures weights of criteria, and the second type measures the amount of deviation from criteria with respect to final goal.

For example form end user's viewpoint we ask about selection, execution and utilization stages as below;

Selection stage:

What is the amount of deviation in selection stage, considering end user satisfaction, from point of view of criteria 1, 2 and 3 ? (Meeting needs of End User)

- Organizational needs

- Economic needs

- Social needs

What is the relative importance of criterion 1, 2 and 3, considering End user satisfaction? (Meeting needs of End User)

- Organizational needs

- Economic needs

- Social needs

Execution stage:

What is the amount of deviation during Execution stage, from the goals set at the end of selection stages, from viewpoint of Criteria 1, 2 and 3?

- Cost

- Time

- Scope

What is the relative importance of criterion 1 and 2, considering End user satisfaction?

- Cost

- Time

- Scope

Utilization stage:

What is the amount of deviation during Utilization stage, from the goals set at the end of execution phase, from viewpoint of Criteria 1,2...8?

- Usefulness

- Functionality

- Meeting requirement

- Perceived value

- Operating cost

- Maintenance cost

- Impact on End user 
- Easy to use

What is the relative importance of criterion $1,2 \ldots 8$, considering End user satisfaction?

- Usefulness

- Functionality

- Meeting requirement

- Perceived value

- Operating cost

- Maintenance cost

- Impact on End user

- Easy to use

After gathering the data it is time to calculate $a_{1}, a_{2}$, and $a_{3}$. We will explain for selection stage, and other two deviations have similar procedure. Having three criteria for measuring deviation from the goals occurred during selection stage, we have three weights, $W_{1}, W_{2}$ and $W_{3}$, with two constraints:

1) $W_{1}+W_{2}+W_{3}=1$

2) $0 \leq W_{1}, W_{2}, W_{3} \leq 1$

On the other hand, we have three numbers that show the amount of deviation in each of the three criteria. These numbers $\left(C_{1}, C_{2}\right.$ and $\left.C_{3}\right)$ are numbers between 0 and 5 .

Now the weighted summation of deviation is calculated by multiplying $W_{1}, W_{2}$ and $W_{3}$ to its corresponding criteria $C_{1}, C_{2}$ and $C_{3}$. Then we have a number which could be easily approved that is between 0 and 5;

$$
\begin{aligned}
& 0 \leq W_{1}, W_{2}, W_{3} \leq 1 \\
& 0 \leq C_{1}, C_{2}, C_{3} \leq 5 \\
& 0 \leq W_{1} C_{1}+W_{2} C_{2}+W_{3} C_{3} \leq 5
\end{aligned}
$$

This amount is indeed weighted amount of deviation in selection stage. We label it as $X_{1}$ as below;

$$
0 \leq X_{1} \leq+5
$$

To use this amount in our model we need to translate it to the radian or degree using below formula;

$$
a_{1}=(180 / 5) \times X_{1}
$$

Therefore the amount of $a_{1}$ would be a degree between 0 and $+180$

$$
0 \leq a_{1} \leq+180
$$

Applying same procedure for $a_{2}$ and $a_{3}$ we could easily calculate $a_{2}$ and $a_{3}$ based on their own criteria;

$$
\begin{aligned}
& 0 \leq a_{2} \leq+180 \\
& 0 \leq a_{3} \leq+180
\end{aligned}
$$

Following this procedure all the relative importance i.e. $i_{1}$, $i_{2}$ and $i_{3}$, and all amounts of deviation from the goal i.e. $a_{1}, a_{2}$ and $a_{3}$ are calculated. Now we could calculate the estimated amount of total deviation $\mathrm{D}$, using these numbers.

\subsection{Error of the Model}

All models are a face of reality not exact state of it. The circles model is also a picture of what really happens and it has some error. To verify a model at we should show the error of the model to ensure that all the formulas used in the model are matched to the model elements. The model uses two main formulas:

$$
\begin{aligned}
& D=\left(i_{1}+i_{2}+i_{3}\right) a_{1}+\left(i_{2}+i_{3}\right) a_{2}+i_{3} a_{3} \\
& D=i_{1} a_{1}+i_{2} a_{1}+i_{3} a_{1}+i_{2} a_{2}+i_{3} a_{2}+i_{3} a_{3}
\end{aligned}
$$

In these formulas there are six parameters i.e. $a_{1}, a_{2}, a_{3}, i_{1}$, $i_{2}$ and $i_{3}$. In Appendix we mathematically prove that these six parameters are arcs and radiuses in the model and since combined together estimate the final arc (D). This estimation has a specific amount of error that is low for lower amounts of deviation. In Appendix we show that for deviations from 0 to 45 degrees the amount of error is low enough to we neglect it. But for deviations more than 45 degrees it is not suitable because of higher amounts of errors. It should be noted that this limitation is only for the amount of deviation in execution and utilization stages. And the deviation of the first stage i.e. selection, never creates any error.

\subsection{Different Situations}

The model could be applied for a variety of situations with different input parameters. We have six main inputs, relative importance $i_{1}, i_{2}$ and $i_{3}$, and deviations, $a_{1}, a_{2}$ and $a_{3}$. To check functionality of our model due to the changing of the inputs i.e. $i_{1}, i_{2}$ and $i_{3}$, we illustrate three extraordinary cases.

The figures $2 \mathrm{a}, 2 \mathrm{~b}$ and $2 \mathrm{c}$ show these three cases where $i_{2}, i_{3}<<i_{1}, i_{1}, i_{3}<<i_{2}$ and $i_{1}, i_{2}<<i_{3}$ respectively. To investigate only the effect of $i_{1}, i_{2}$ and $i_{3}$, we set the $a_{1}=a_{2}=a_{3}=15$ degrees, to eliminate their influence. As it can be seen from the figures, the model predicts different levels of success (output) for different levels of $i_{1}, i_{2}$ and $i_{3}$. Fig 2 shows us the applicability of the model for various inputs in different situations.
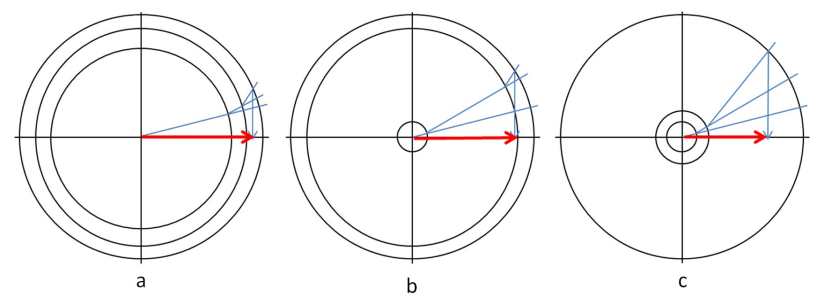

Fig. 2 The Effect of Changing Relative Importance

To check the outputs of the model based on the changes of other inputs, i.e. $a_{1}, a_{2}, a_{3}$, we set $i_{1}=i_{2}=i_{3}=1 / 3$. This is due to omit the effects of changes in $i_{1}, i_{2}$ and $i_{3}$. We show five different statuses in Fig 3, happening because of the variation of the inputs. Obviously the model supports a diverse range of possible situations in which a project could be fully failure $(\mathrm{S}=-1)$ until fully successful $(\mathrm{S}=1)$.

\section{Application of the Model}

To test the model applicability on the real projects we need to bring an example. Consider a bridge construction project aim to connect two sides of a river inside a city. It has been selected not 


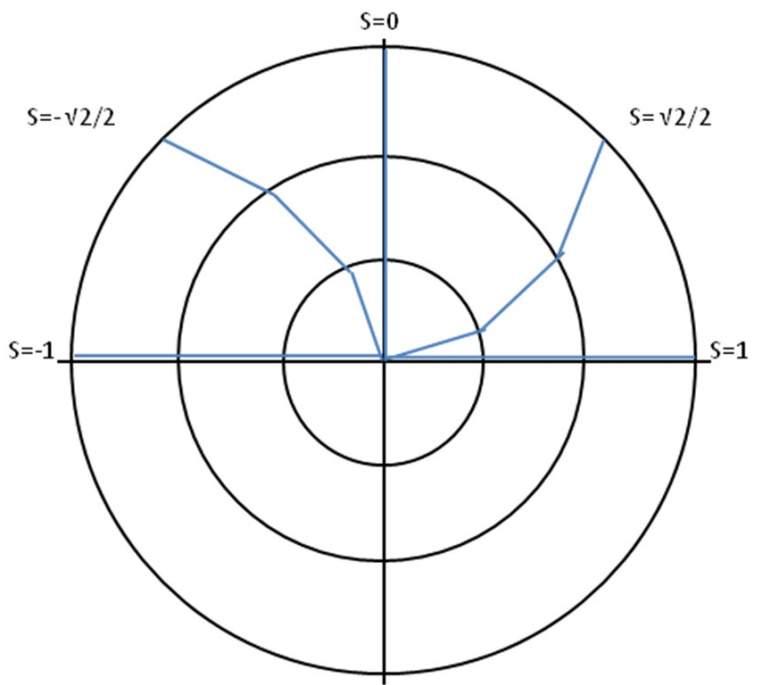

Fig. 3 The Effect of Changing Deviations

very well whereas there were some other ways to connect the river' s sides. Unfortunately the local government as the client of project has selected it without any comprehensive investigation because of the political pressures. The first deficiency of the bridge is its improper location that is far from the center of the traffic load of the city. The second failure of the project is narrow width that is because of poor design so that it could not satisfy the amount of traffic load. But its construction was completed precisely as it planned, by an experienced international construction company. It is going to be used by citizens without any supplemental customer service from local government, and therefore this would be the third lack of the project if not revised in the future (during utilization stage). The aim is to evaluate and predict project success for different situations. Keeping in mind that in this example design and construction are part of execution stage.

Although the financial supporter of the project is local government but citizens are its real payers due to the taxes they pay every year. From end users' viewpoint (citizens), this project was not selected and executed suitably. It has huge deviations from their objectives despite the fact that it is constructed on time, under budget and in the best quality using high technologies. Inappropriate selection not only has a direct impact $\left(D_{12}\right)$ on deviation from satisfaction of end users but also indirectly increase deviation via increase of $D_{12}$. This bridge consumes a massive amount of money from pockets of citizens during its execution. And therefore it has not only solved the main problem of the citizens (connecting the two sides of the river, $0<D_{1}$ ) but also it has wasted lots of money in the false direction that is kind of influenced deviation $\left(0<D_{12}\right)$. In this case the extent to which the project selection is inappropriate $\left(a_{1}\right)$, and also the extent to which its construction process consumes more resources such as money, time and materials $\left(i_{2}\right)$, the amount of influenced deviation $D_{12}$ will increase. In other word $D_{12}$ is a function of $a_{1}$ and $i_{2}$.

Similar proof could be applied to ascertain the two other influenced deviations $\left(D_{13}, D_{23}\right)$. In our example the meaning of $D_{13}$ (influenced deviation during utilization stage) would be kind of consuming more gasoline and time to pass the river because of the farness and inappropriate location of the bridge. It is obvious that $D_{13}$ is a function of $a_{1}$ and $i_{3}$. The higher $a_{1}$ will consume the resources more unsuitably during utilization stage And the higher $i_{3}$ will result in higher resource consumption.

The $D_{23}$ is not zero because the amount of deviation from goals in execution stage is not zero $\left(0<a_{2}\right)$. If we have some amount of deviation in second stage, for instance because of poor design and narrow width of bridge, the citizens will experience huge traffic during utilization stage especially in rush hours. This influenced deviation, $D_{23}$, would be a function of $a_{2}$ (how much it is narrow due to poorly designed) and $i_{3}$ (how much traffics in utilization stage increases and consumes time of citizens on the streets), which result in $a_{2} i_{3}$ (how much its narrow width will suffer the citizens during utilization stages). This effect is different than $D_{12}$ and $D_{13}$.

Now we calculate and predict the amount of success of project, assuming that the local government will not do any supplemental project to connect the bridge to the traffic centers of the city and therefore deviation in the future in the utilization stage will happens.

The schematic scheme of this specified project is shown in the Fig 4 in which the model outputs are calculated based on the inputs;

Inputs:

- $i_{1}=i_{2}=i_{3}=1 / 3$ (judged by experts)

- $a_{1}=\pi / 3$ (inappropriate selection of the project)

- $a_{2}=\pi / 4$ (inappropriate design of the project)

- $a_{3}=\pi / 4$ (inappropriate supplemental customer services)

Outputs: Pure deviation:

- $D 1=a_{1} i_{1}=\pi / 9$ (direct deviation caused by poor selection stages)

- $D 2=a_{2} i_{2}=\pi / 12$ (direct deviation caused by poor execution stage)

- $D 3=a_{3} i_{3}=\pi / 12$ (direct deviation caused by poor utilization stage)

Influenced deviation:

- $D_{12}=a_{1} i_{2}=\pi / 9$

- $D_{13}=a_{1} i_{3}=\pi / 9$

- $D_{23}=a_{2} i_{3}=\pi / 12$

Total deviation:

$D=D_{1}+D_{2}+D_{3}+D_{12}+D_{13}+D_{23}=7 \pi / 12 \simeq 105$ degrees Total success:

$S=\operatorname{Cos}(D) \simeq-0.25$

One important point is that pure deviation during utilization stage $\left(D_{3}\right)$ is because of two main issues:

- Malfunctions or unsatisfying the users' requirements (for example even bridge is strong in front of wind, earthquake or automobiles' load, but its width is small and creates a heavy traffic on the bridge during rush hours)

- Doing after sale customer services weakly (for example local government should do other operations such as maintenance, recovery or even construction of other roads to 


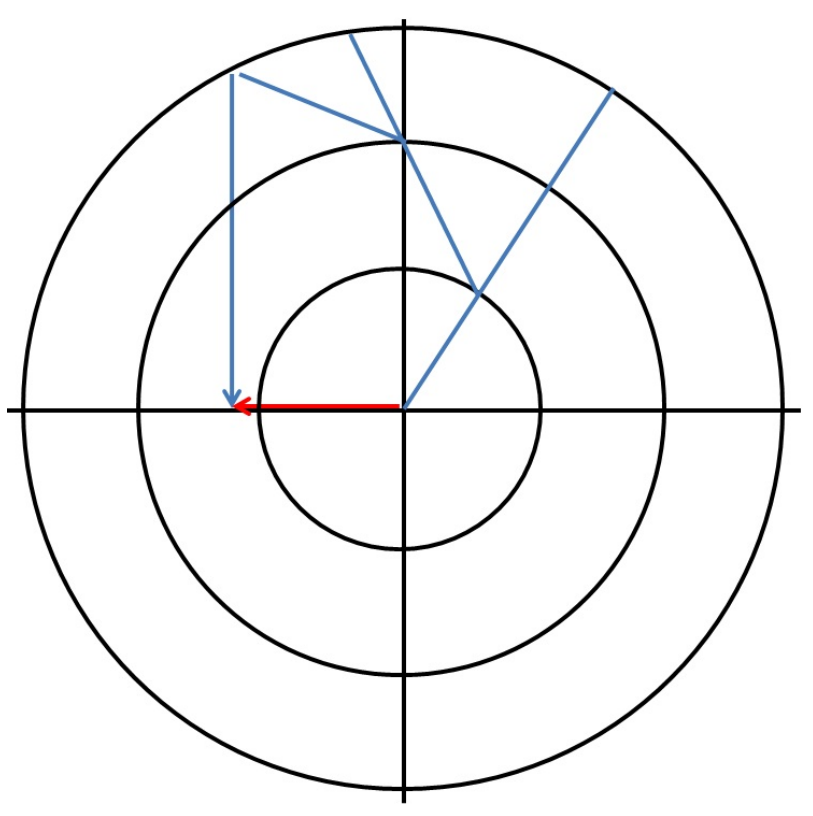

Fig. 4 Success of Bridge Construction Project

facilitate the accessibility of the bridge). As mentioned before although the bridge is far from the main traffic centers, but local government could connect these traffic centers to entrance edges of the bridges to reduce dissatisfaction due to its farness. Unfortunately local government has not done these activities.

Indeed client not only should be worry about functionality of the final product but also should do some other activities even after delivery of the final product to prevent more deviation from end users' satisfaction. These supplementary activities depend on the type of the project and its specific conditions. In our example both of the above mentioned lacks happened and therefore we have a high pure deviation during utilization stage.

Forgetting such additional activities during utilization stage is a significant lack in measuring project success in project management discipline. Using Circles model we would be able to take into account this phenomena in a quantitative manner.

\section{Discussion}

The model we proposed in this paper tries to quantify the success of project. It maps the amount of success onto a number between -1 and 1 . This is quite useful for project manager or owner to see or to predict the performance of its project quantitatively. They also could analyze the performance of each stage and relationship between these performances. This is possible through analysis of the influenced deviations $\left(D_{12}, D_{13}\right.$ and $\left.D_{23}\right)$. After applying the methods introduced in previous steps we could calculate pure deviations $\left(D_{1}, D_{2}\right.$ and $\left.D_{3}\right)$ alongside influenced deviations. Then we could draw a diagram same as fig 5 , and we could analysis the behavior of different types of project in different situations. Especially the performance of each stage as a function of performance of previous stages. In this light a useful approach to analysis the system behavior is what if analysis through observing outputs by changing the inputs $\left(i_{1}, i_{2}\right.$, and $i_{3}, a_{1}, a_{2}$ and $\left.a_{3}\right)$. This will allow us to see the success of the project in different circumstances. Afterward the sensitivity analysis could be applied to explore the sensitivity of the success of project to different deviations in different stages with different relative importance. This would be a direction for future studies and it would help us to know more about the nature of project as a unique activity.

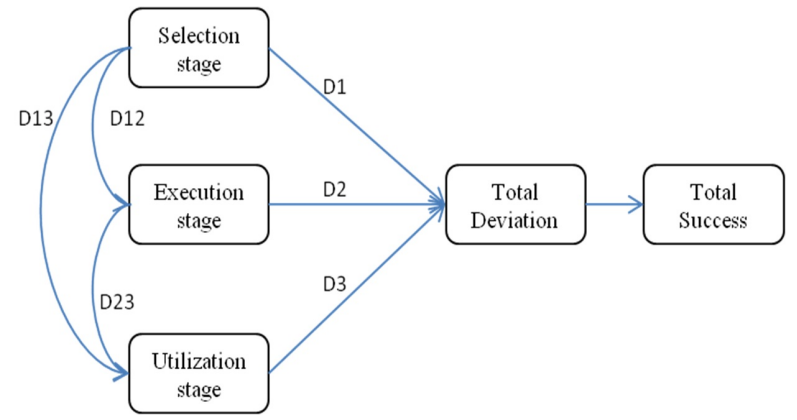

Fig. 5 Exploring Relationship between stages Using the Circles Model

We assume that the amount of deviations $a_{1}, a_{2}$ and $a_{3}$ are positive or zero. There are situations in which project is very high performance and as a result it exceeds its goals. This kind of projects have negative deviations $\left(a_{1}, a_{2}\right.$ and $\left.a_{3}<0\right)$. This situation is also applicable through Circles model but it needs more investigations.

It is generally agreed that measuring project success is a vague area (Hyvari, 2006). The vagueness is partially due to the existence of different stakeholders in a project. Each stakeholder has its own objective from the project. These objectives are almost different or even contradictory. In this paper we consider one of stakeholders, i.e. End user, and try to measure success of project from its view point. We set the criteria for measuring deviations based on its satisfaction. Although our main concern in this paper is end user, but the model has flexibility to measure success of the project from point of view of other stakeholders such as client, project team, suppliers and etcetera. The key point is adapting the model with corresponding stakeholder. The adaptation process could be easily done only by changing the criteria that we mentioned in section 2 . In other word the main goal of all criteria would be satisfaction of specified stakeholder that we aim to measure project success from its viewpoint. And therefore we do not have a single amount of success for a project. For different stakeholder we could determine different magnitude of success. It makes sense because each stakeholder has its own criteria to evaluate project success. In the real world this conditions happens frequently, when in a project, different stakeholders have different understanding of project success.

It is also useful to be mentioned that the Circles model has potential to be applied on measuring program and portfolio success. In this article we only discuss the single project and its evaluation. There are conditions where plenty of projects are interconnected to make a program or above that to make a portfolio of projects. We need to do more investigations about capability of the model to be extended and applied on the multi project domain.

\section{Conclusion}

We introduced a model for measuring project success in an integrated manner. To measure project success we should take into account all the life cycle i.e. selection, executions until utilization. Each project has a final goal and during each of three stages there are some deviations from the goal happening 
because of the managerial or functional mistakes. The extent to which project managers or generally project decision makers make mistakes, the project deviate more and more from its final goal. We used the concept of deviation to measure the amount of reaching the goals or in other word the amount of success. The Circles model has a high valid face model because its nature is similar to the concept of deviations from the goal. Although the model has a specific amount of error corresponding to the amount of deviations, but still it works with negligible error even for the higher amount of deviation. We showed that the model could be applied for a diverse range of project with different amount of deviations and relative importance of the stages. We also applied it in a quantitative example to illustrate its application to the real world.

In addition to the above-mentioned advantageous the model has four other potential opportunities to be extended. In fact these four extensions are four directions for future studies. The first extension is deliberating on the model elements and finding relationship between performances of stages. Prior stages have a functional effect on the next stages and this could be illustrated through influenced deviations. But still it needs more investigations to analyze under which circumstances and in which kind of projects these influences are high. The second extension is to apply the model for measuring high performance projects in which not only do not have any deviation but also exceed the project goals. In this condition the amount of deviation in one or more than one of the stages is negative amount. The third extension is about multi stakeholder characteristic of the projects. Each project has different stakeholders and each stakeholder has its own goal in the project. To measure project success from different viewpoints, it is enough to set measuring criteria and the final goal based on the satisfaction of corresponding stakeholder. And thus the model will measure the success from point of view of that stakeholder which might be different from end user viewpoint. The forth extension extents the model to a multi project domain in which there are several project interrelated under a big program or portfolio. This application needs more investigations to rationally connect result of different projects and construct the whole program or portfolio success.

\section{Appendix}

The model has a specific amount of error because of noncentrality of three arcs corresponding to three stages. Here we aim to prove that the amount of error is negligible for lower amounts of deviation. To show this we bring an example in which $i_{1}=i_{2}=i_{3}=1 / 3$ and $a_{1}=a_{2}=0$ and $0<a_{3}$. This is because we aim to illustrate only the effect of increasing $a_{3}$ on increasing the amount of error. This specific situation is shown in the Fig A.1. In this figure the exact amount of deviation is $\mathrm{d}$ but the formulas of the model calculate it as D and D always is lower than $\mathrm{d}(D<d)$.

$$
\begin{aligned}
& D=\left(i_{1}+i_{2}+i_{3}\right) a_{1}+\left(i_{2}+i_{3}\right) a_{2}+i_{3} a_{3} \\
& D=i_{1} a_{1}+i_{2} a_{1}+i_{3} a_{1}+i_{2} a_{2}+i_{3} a_{2}+i_{3} a_{3}
\end{aligned}
$$

Therefore the amount of error of the model is difference between $\mathrm{d}$ and $\mathrm{D}$;

$$
\text { Error }=d-D
$$

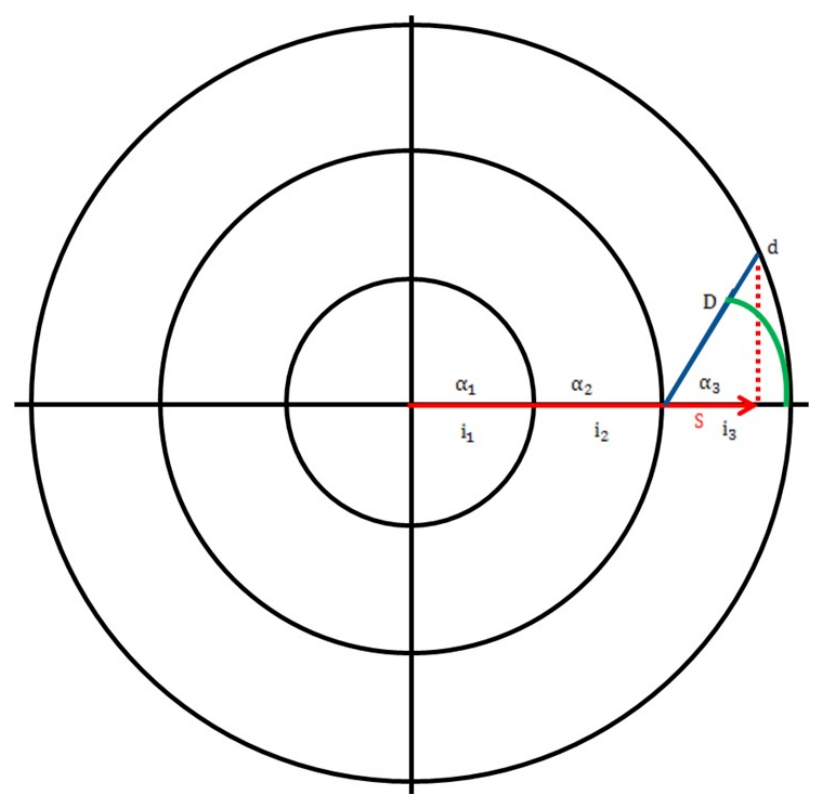

Fig. A.1 Error of the Model due to Non-Centrality

Visually it can be seen that the lower amount of $a_{3}$ the lower error of the model. The extent to which $a_{3}$ grows, the amount of error grows. To quantify this concept, using simple mathematics and geometry, we calculated d. Afterward we found the amount of error for different amount of $a_{3}\left(0<a_{3}<60\right.$ degrees) resorting MATLAB software. The results are shown in the Fig A.2. It can be seen that even for higher amounts of deviation ( $a_{3}=60$ degrees), the amount of error is negligible. It should be emphasize that for lower deviations the accuracy of the model is more.

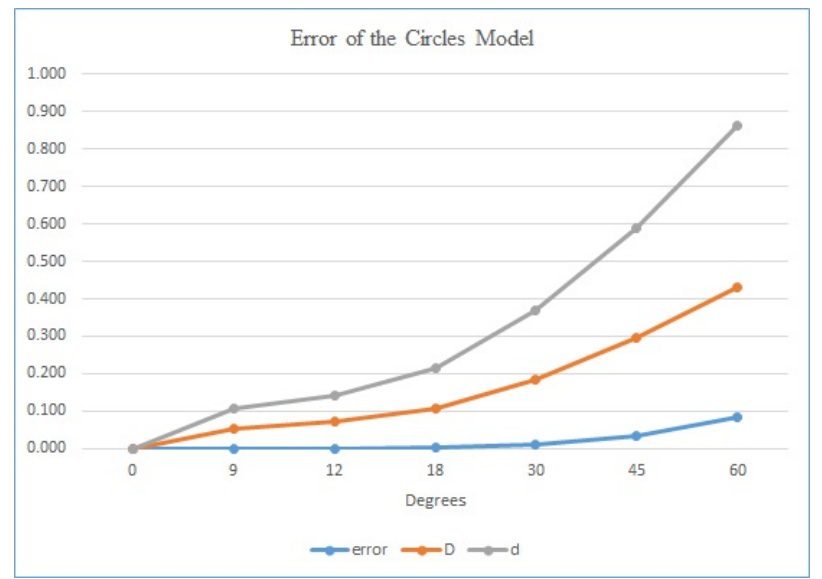

Fig. A.2 Error of the Model based on different deviations

This process could also be done for $a_{2}$ and even for the case in which both $a_{2}$ and $a_{3}$ are not zero. And the process of calculating error for them is same as the process we discussed here for $a_{3}$. For each case the lower the deviation would result the lower the error of the model.

It must be mentioned that the error of the model is only due to the $a_{2}$ and $a_{3}$ not $a_{1}$, because $a_{1}$ is an angle that its core is center of the circles. And all terms relating to the $a_{1}\left(i_{1} a_{1}\right.$, $i_{2} a_{1}$ and $\left.i_{3} a_{1}\right)$ are correct without any error. On the other word the model could accept any amount of $a_{1}$ even higher amounts without increasing any error. While about $a_{2}$ and $a_{3}$, the error of the model is sensitive to their amplitude. 


\section{References}

[1] Atkinson, R., 1999. Project management: cost, time and quality, two best guesses and a phenomenon, its time to accept other success criteria. International Journal of Project Management 17, 337-342.

[2] Baccarini, D., 1999. The logical framework method for defining project success.Project Management Journal 30, 25-32.

[3] De Wit, A., 1988. Measurement of project success. International Journal of Project Management 6, 164-170.

[4] Hyvari, I., 2006. Success of project in different organizational conditions, Project Management Journal, 37 (4), 31 ?41.

[5] Ika, L.A., 2009. Project success as a topic in project management journals. Project Management Journal 40 (4), 6-19.

[6] Jugdev, K., Muller, R., 2005. A retrospective look at our evolving understanding of project success. Project Management Journal 36, 19-31.

[7] Khang D.B., Moe T.L., 2008. Success Criteria and Factors for International Development Projects: A Life Cycle-based Framework. Project Management Journal 39 (1), 72-84.

[8] Munns, A.K., Bjeirmi, B.F., 1996. The role of project management in achieving project success. International Journal of Project Management 4, 81-87.

[9] Parsanejad M.R., Matsukawa H., 2012. Measuring project success; Toward a Comparative Framework. International Symposium on Reliability Engineering and Risk Management. Kanagawa University, Japan.

[10] Pillai, A. S., Joshi, A., Rao, K.S., 2002. Performance measurement of $\mathrm{R}$ and $\mathrm{D}$ projects in a multi-project, concurrent engineering environment. International Journal of Project Management 20,165-177

[11] PMBOK, 2008. A guide to the project management body of knowledge guide. Project Management Institute, USA.

[12] P2M, 2003. Project and Program Management for Enterprise Innovation. Project Management Association of Japan, Japan.

[13] Yu, A. G., Flett, P. D. and Bowers, J. A., 2005. Developing a Value-Centered Proposal for Assessing Project Success?, International Journal of Project Management, 23(6) 428-436.

\section{Mohammadreza PARSANEJAD}

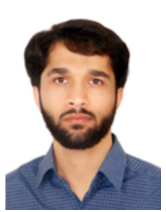

is now $\mathrm{PhD}$ candidate in Keio University. He received his B.E. degree from Iran University of Science and Technology and his M.Sc from Tehran University. His research interest includes performance measurement of projects and supply chains.

\section{Hiroaki MatsukaWa}

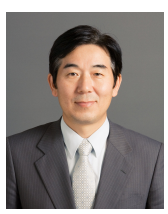

is a professor at Keio University. He received his PhD degrees from Tokyo Institute of Technology, Tokyo, Japan, in 1992. Main research interests include production \& inventory control and supply chain management (SCM). Continuous effort is dedicated to clarify principles of management on those research topics such as scheduling, manufacturing strategy, project management and other topics related to production and logistics. Quantitative methods were frequently applied for solving management problems. Recent interests include supply chain risk management under disruptive event, and energy supply control applying supply chain optimization approaches.

\section{Tomoichi SATo}

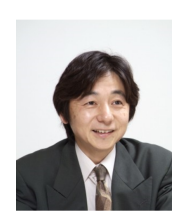

is General Manager of ICT Management Office at JGC Corporation in Yokohama, Japan. He is also Lecturer at Keio University, University of Tokyo and Hosei University in Tokyo, Japan. He finished his master in Chemical Engineering at University of Tokyo in 1982. From 1982 to present, he has been working for JGC Corporation as process systems engineer and later as project planner/manager mainly in the oil \& gas industry. He obtained Doctoral degree from University of Tokyo in 2010 by researches on project management theory. His interests include project management, supply chain management and risk analysis.

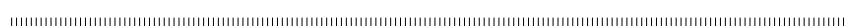

